\section{Vacina antipneumocócica conjugada e polissacarídea}

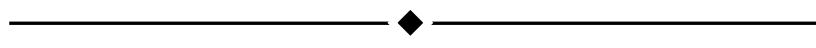

Prezado Editor,

Em consideração ao artigo publicado por Di Nuzzo \& Fonseca ${ }^{1}$, gostaríamos de esclarecer que as informações publicadas referentes às imunizações contra doenças pneumocócicas necessitam retificações, conforme exposto a seguir.

No referido artigo, são apresentadas quatro vacinas pneumocócicas conjugadas. Entretanto, no Brasil, existe apenas uma apresentação de vacina pneumocócica conjugada, a saber, a vacina 7-valente conjugada com o toxóide diftérico CRM197 (sorotipos 4, 6B, 9V, 14, 18C, 19F, 23F), estando indicada para administração a partir dos 2 meses de idade (e não apenas entre 2 meses e 2 anos), podendo ser aplicada até os 5 anos de idade. Crianças que iniciam a vacinação com a vacina 7 -valente podem receber posteriormente uma dose da vacina 23 -valente como complementação em casos especiais (alto risco) 2,3.

Crianças imunodeprimidas acima de 2 anos de idade devem receber duas doses da vacina 7-valente (com 2 meses de intervalo) e uma dose da vacina 23-valente (2 meses após a última dose da 7-valente).

Estudos recentes sobre a eficácia e a imunogenicidade da vacina para pacientes prematuros, assim como para crianças maiores necessitando proteção contra doenças não-invasivas (exemplo: otite média aguda), têm servido de referência para ampliar as indicações e a população-alvo dessa vacina ${ }^{4-7}$.

Quanto à vacina 23-valente, deve-se classificá-la entre as vacinas polissacarídeas, compostas de antígenos de polissacarídeo capsular purificado de 23 sorotipos, estando disponível em nosso meio somente para administração a partir de 2 anos de idade.

As vacinas conjugadas 9 e 11 -valente ainda não estão disponíveis para comercialização, encontrando-se em fase final de pesquisa.

\section{Roberto Valdez}

Diretor Técnico da Previne Centro de Vacinação, Porto Alegre, RS

\section{Everton Sukster}

Diretor Técnico da Imune Clínica de Vacinação, Porto Alegre, RS

\section{Referências}

1. Di Nuzzo DV, Fonseca SF. Anemia falciforme e infecções. J Pediatr (Rio J). 2004;80:347-54.

2. Centers for Disease Control and Prevention. Prevention of pneumococcal disease among infants and young children: recommendations of the Advisory Committee on Immunization Practices. MMWR. 2000;49(No. RR-9).

3. Centers for Disease Control and Prevention. Recommended childhood and adolescent immunization schedule-United States, July-December 2004. MMWR. 2004;53:Q1-3.

4. Black S, Shinefield H, Fireman B, Lewis E, Ray P, Hansen JR, et al. Efficacy, safety and immunogenocity of heptavalent pneumococcal vaccine in children. Pediatr Infec Dis J. 2000;19:187-95.

5. Eskola J, Kilpi T, Palmu A, Jokinen J, Haapakoski J, Herva E, et al. Efficacy of a pneumococcal conjugate vaccine against acute otite media. N Engl J Med. 2001;344:403-9.

6. Shinefield H, Black S, Ray P, Fireman B, Schwalbe J, Lewis E. Efficacy, immunogenicity and safety of heptavalent pneumococcal conjugate vaccine in low birth weight and preterm infants. Pediatr Infect Dis J. 2002;21:182-6.

7. Whitney CG, Farley MM, Hadler J, Harrison LH, Bennett NM, Lynfield $R$, et al. Decline in invasive pneumococcal disease after the introduction of protein-polysaccharide conjugate vaccine. $\mathrm{N}$ Eng J Med. 2003;348:1737-46.
Senhor Editor,

Foi com grande interesse que analisamos o artigo de Rocha Filho et al. ${ }^{1}$, e sobre ele gostaríamos de tecer os seguintes comentários e indagações:

1) Nos espaçadores de grande volume, as partículas respiráveis permanecem por maior tempo em suspensão antes de serem inaladas ou decantarem no dispositivo; sendo assim, para um melhor aproveitamento da droga, é recomendável que o período de inalação seja necessariamente superior àquele dos seus congêneres de pequeno volume, em geral 30 segundos, e não 10 segundos, como mencionado no artigo.

2) Como pré-escolares de 3 anos têm uma dinâmica respiratória distinta de escolares de 7 anos, parece-nos mais adequado fazer análises de deposição igualmente distintas, por faixa etária, e não incluí-los na mesma análise; qual teria sido a média de idade desses dois grupos? 
3) Já que se trata de um teste de hipóteses, deixa-se de incluir, nas referências que se faz ao tamanho amostral, o tipo e a quantidade da diferença que se quer testar; teriam sido diferenças entre médias ou proporções de deposição pulmonar? Que valores foram tomados? Em se tratando de diferença entre médias, qual foi o valor da variância pooled que fundamentou o cálculo amostral?

4) Na falta dessas premissas - indispensáveis para a análise do poder do estudo -, servimo-nos da fórmula matemática aplicável às diferenças entre médias e chegamos à aproximação do tamanho amostral necessário para se assegurar o power de $80 \%$ explicitado na metodologia; assim, para se detectar diferenças entre médias de deposição entre Inalair ${ }^{\circledR}$ e Flumax ${ }^{\circledR}$, seriam necessários, idealmente, cerca de 20 crianças e em torno de 34 adultos $^{2}$. Sendo assim, cabe-se indagar: qual o verdadeiro poder do estudo?

5) É recomendável que o valor de $\mathrm{p}$ venha acompanhado dos limites superior e inferior do intervalo de confiança a $95 \%$, permitindo, assim, a real compreensão da significância estatística e sua conseqüente interpretação do ponto de vista clínico; quais teriam sido esses valores? Como a análise da Figura 2 sugere superposição das variações de deposição pulmonar entre os espaçadores testados, esses resultados mereceriam maior aprofundamento na discussão? Qual seria a validade interna e externa da investigação? Os resultados obtidos permitem afirmar que "nosso estudo demonstra claramente que os espaçadores de pequeno volume são superiores aos de grande volume"?

6) Ademais, no ensaio clínico randomizado incluído nas referências bibliográficas ${ }^{3}$, o espaçador de grande volume testado foi uma garrafa de água mineral de $500 \mathrm{ml}$, que se mostrou tão eficaz quanto o nebulizador no tratamento da asma aguda, mesmo que $95,8 \%$ dos pacientes estudados tenham sido pré-escolares e escolares; espaçadores de grande volume são sempre menos eficazes nessas faixas etárias?

7) Como a imprecisão é indissociável de qualquer técnica semiquantitativa, a deposição de tecnécio fitato per se corresponderia à proporção efetivamente inalada de broncodilatadores e/ou corticóides cujas moléculas foram ou não marcadas com isótopos radioativos? Quais seriam as reais limitações dos estudos de deposição semiquantitativos realizados in vivo? Deveriam esses aspectos ser aprofundados na discussão?

8) Entendemos, ademais, que a noção de carga eletrostática dos espaçadores plásticos mereceria também maior detalhamento: diferentes tipos de plástico reúnem a mesma carga eletrostática? Flumax ${ }^{\circledR}$, fabricado com PVC, comportar-se-ia da mesma forma que Aerochamber ${ }^{\circledR}$ e espaçadores feitos com policarbonato? Há métodos consagrados na literatura para reduzir essa carga eletrostática? Qual o papel dos detergentes caseiros nesse caso? As diferenças de deposição entre espaçadores de metal e plástico reduzem ou aumentam quando estes últimos são enxaguados com aqueles produtos? Seria metodologicamente recomendável enxaguar os espaçadores de plástico testados antes de se analisar a deposição pulmonar?
9) Já que nosso estudo foi citado (referência número 10), vale também acrescentar que, como parte inseparável do estudo de deposição semiquantitativo que fizemos com o Flumax ${ }^{\circledR}$, incluímos a avaliação clínica e, nela, obtivemos resultados favoráveis mesmo que a idade média das crianças estudadas tenha sido de 5,5 anos; como explicar essa resposta já que espaçadores de grande volume devem ser reservados a adolescentes e adultos? Esse achado deveria ou não ser incluído na discussão do artigo ora comentado?

10) O que interessa aos clínicos é aquilo que se passa na vida real, e isso pode ser analisado em estudos de efetividade; em dois diferentes estudos que avaliaram o programa de asma de Belo Horizonte, no qual as crianças usaram exclusivamente o espaçador de grande volume da marca Flumax ${ }^{\circledR}$, foram avaliadas $2.141^{4}$ e cerca de $700^{5}$ crianças, das quais $75 \%$ eram menores de 5 anos de idade, obtendo-se, respectivamente, uma redução de 75,8 e $89 \%$ na taxa de hospitalização e de 85 e $91 \%$ nas visitas a serviços de pronto-atendimento; como, então, explicar esses resultados numa população constituída basicamente por pré-escolares?

11) Finalmente, de acordo com Anderson 6 , "there is no 'best' outcome or 'gold standard' in the assessment of inhaled drug delivery... We need more trials comparing in vitro with in vivo outcomes...; in the final analysis, however, there is no substitute for clinical trials in patients". Caberia aprofundar a discussão desses aspectos no artigo?

\section{Paulo Camargos}

Professor titular, Departamento de Pediatria, Faculdade de Medicina, Universidade Federal de Minas Gerais (UFMG), Belo Horizonte, MG

\section{José Augusto Rubim}

Professor assistente, Departamento de Pediatria, Faculdade de Medicina, Universidade Federal de Minas Gerais (UFMG), Belo Horizonte, MG

\section{Referências}

1. Rocha Filho W, Noronha VX, Senna SN, Simal CJ, Mendonça WB. Avaliação da influência da idade e do volume do espaçador na deposição pulmonar de aerossóis. J Pediatr (Rio J). 2004;80: 387-90.

2. Hulley SB, Cummings SR, Browner WS, Grady D, Hearst N, Newman TB. Designing clinical research: an epidemiological approach. 2nd ed. Philadelphia: Lippincott Williams \& Wilkins; 2001.

3. Duarte M, Camargos P. Efficacy and safety of a home-made nonvalved spacer for bronchodilator therapy in acute asthma. Acta Paediatr. 2002;91:909-13.

4. Fischer GB, Camargos PA. Paediatric asthma management in developing countries. Paediatr Respir Rev. 2002;3:285-91.

5. Fontes MJ. Síndrome sibilante e asma brônquica: proposta de tratamento em crianças e adolescentes através da parceria SUS/UFMG [tese]. Belo Horizonte, MG: Universidade Federal de Minas Gerais; 2002.

6. Anderson PJ. Assessment end points for inhaled drug delivery. Respir Care. 2000;45:737-55. 


\section{Resposta do autor}

\section{Prezado Editor,}

Agradecemos o interesse dos Profs. Camargos e Rubim pelo nosso estudo ${ }^{1}$ e a gentileza de apresentar suas opiniões. Gostaríamos de lembrar que nosso estudo teve como objetivo avaliar a deposição pulmonar de aerossol marcado com isótopos radioativos. Portanto, os dados apresentados não permitem tirar qualquer conclusão em relação à eficiência clínica dos espaçadores estudados. Concordamos com a afirmação de que a deposição pulmonar varia de acordo com a medicação utilizada. No entanto, não encontramos respaldo na literatura para a afirmação de que as partículas respiráveis permanecem por maior tempo em suspensão em espaçadores de grande volume, sendo recomendável um maior período de inalação. Por que 30 segundos e não 20, 25 ou 15 segundos?

É nossa opinião que os principais fatores relacionados com a deposição pulmonar de um determinado aerossol são o tamanho das partículas geradas, mais especificamente o diâmetro médio de massa dessas partículas, e o fluxo inspiratório. Quando se utilizam espaçadores, além das variáveis acima, a carga eletrostática e o volume corrente do paciente (10 a $15 \mathrm{ml} / \mathrm{kg}$ ) passam a ser de fundamental importância. Portanto, uma criança de $15 \mathrm{~kg}$ terá um volume corrente em torno de 120 a $150 \mathrm{ml}$. Bastariam duas incursões respiratórias no espaçador de pequeno volume e cinco incursões no espaçador de grande volume para que todo o conteúdo do espaçador fosse inalado. De fato, um dos co-autores do nosso estudo (Simal CJR) verificou que, independentemente da idade, a quase totalidade do aerossol com isótopos radioativos era inalada nas duas primeiras incursões respiratórias (comunicação pessoal). Trata-se do mesmo co-autor do estudo de Rubim et al. ${ }^{2}$, utilizando técnica idêntica de avaliação da deposição pulmonar. Portanto, não acreditamos que 30 segundos de inalação nos espaçadores de grande volume traria algum benefício adicional.

A carga eletrostática também influencia de forma considerável a deposição pulmonar. Concordamos que a carga eletrostática pode variar de acordo com o material utilizado. É provável que um espaçador de PVC, $\operatorname{como}$ o Flumax ${ }^{\circledR}$, tenha uma carga eletrostática diferente de espaçadores de policarbonato, como o Aerochamber ${ }^{\circledR}$. Além disso, a carga eletrostática sofre influência do clima e do volume do espaçador, estando inversamente relacionada à umidade do ar e ao tamanho do espaçador. Portanto, em clima mais seco e em espaçadores de pequeno volume, o efeito da carga eletrostática é maior, diminuindo o número de partículas respiráveis. De qualquer forma, seja qual for a matéria-prima do espaçador, a carga eletrostática será sempre menor no espaçador de alumínio, pois este não possui carga eletrostática. Essa importante diferença pode ser abolida se os espaçadores forem enxaguados com detergente neutro, fato que eliminaria a carga eletrostática dos espaçadores de PVC ou policarbonato. Nosso estudo tinha como objetivo avaliar a influência dessa carga eletrostática nos diferentes espaçadores, e não faria sentido eliminá-la com o uso de detergente. Acreditamos também que os espaçadores de alumínio sejam muito mais práticos de serem utilizados em serviços de pronto-atendi- mento, pois dispensam a lavagem com detergente neutro após a utilização pelo paciente.

O cálculo do poder da amostra baseou-se no fato de que os três grupos de comparação tinham tamanhos iguais a 9, considerando, ainda, um nível de significância igual a 0,05, um desvio padrão comum igual a 8,7 e uma variância das médias igual a 68,756 . Esses dados são necessários para 0 cálculo do poder quando temos três ou mais grupos de comparação. Achamos, ainda, que o comentário referente ao valor $\mathrm{p}$ não procede, uma vez que não foram feitos cálculos de intervalos de confiança. A Figura 2 mostra gráficos boxplot para a deposição pulmonar nos três espaçadores de acordo com a idade, e não intervalos de confiança. Realmente, a questão não é se a diferença entre os três grupos é significativa. Uma diferença pequena pode ser estatisticamente significativa e não ter relevância clínica. O que realmente importa é o tamanho da diferença.

É importante salientar que os espaçadores de grande volume vêm sendo cada vez menos utilizados em nível mundial, e até mesmo no Brasil. Enquanto dispomos de inúmeros espaçadores de pequeno volume, o Flumax ${ }^{\circledR}$ é o único espaçador de grande volume disponível no mercado brasileiro. Os espaçadores de grande volume são bojudos, incômodos para transportar e guardar, considerados por muitos de montagem difícil, além de possuírem carga eletrostática. Soma-se a isso o fato de que, em nenhum momento, documentou-se que espaçadores de grande volume são superiores aos de pequeno volume. Nosso estudo está de acordo com inúmeros outros da literatura que indicam a superioridade de espaçadores de pequeno volume, os quais, ao contrário dos seus congêneres, podem ser utilizados em qualquer idade. No último Congresso Brasileiro de Pneumologia Pediátrica, realizado no Rio de Janeiro em abril de 2004, o Dr. Hawm Tildden, analisando a deposição pulmonar com técnica mais elaborada do que aquela por nós utilizada, confirmou a baixa deposição pulmonar quando testou o Flumax ${ }^{\circledR}$ em seu laboratório na Holanda (comunicação pessoal).

Por fim, estamos de pleno acordo com os Profs. Camargos e Rubim no sentido de que muitas das afirmações feitas por nós e por eles precisam ser respaldadas por trabalhos clínicos bem feitos. A experiência clínica de Belo Horizonte no tratamento da criança asmática em nível de saúde pública é um exemplo de como esses pacientes podem ser beneficiados através de um projeto bem feito e colocado em prática com competência. Apesar da redução importante na taxa de internação por asma, questionamos se a utilização de espaçadores de pequeno volume traria um benefício ainda maior. Essa preocupação parece estar presente na equipe que coordena o projeto, tendo em vista que espaçadores de pequeno volume já estão disponíveis na rede pública de Belo Horizonte. Da mesma forma, o estudo de Rubim et al. não se propôs a comparar a eficácia clínica de diferentes espaçadores. Em nenhum momento inferimos que espaçadores de grande volume são ineficazes, mas sim que os espaçadores de pequeno volume são superiores aos de grande volume no que diz respeito à deposição pulmonar. Mas hipóteses são cantigas de ninar com as quais os mestres embalam seus pupilos. Sem o apoio de estudos clínicos bem elaborados, elas não passam de ficção. Para isso, está em andamento, em nosso serviço, um estudo duplo-cego comparativo, onde avaliare- 
mos cerca de 200 pacientes utilizando diferentes espaçadores. Esperamos, com isso, responder algumas das dúvidas aqui levantadas, pois uma meia verdade pode ser uma mentira inteira. A impressão clínica e a experiência pessoal certamente são válidas para se formar uma hipótese, mas não são substitutas de dados objetivos obtidos através de estudos clínicos bem elaborados. A hipótese é passível de teste, mas até ser testada não deve ser encarada como fato.

\section{Wilson Rocha Filho}

Coordenador do Serviço de Alergia e Pneumologia Pediátrica, Hospital Felício Rocho e Centro Geral de Pediatria, Belo Horizonte, MG

\section{Referências}

1. Rocha Filho W. Noronha VX, Senna SN, Simal CJ, Mendonça WB. Avaliação da idade e do volume do espaçador na deposição pulmonar de aerossóis. J Pediatr (Rio J). 2004;80:387-90.

2. Rubim JA, Simal CJ, Lasmar LM, Camargos PA. Deposição pulmonar de radioaerossol e desempenho clínico verificado com espaçador desenvolvido no Brasil. J Pediatr (Rio J). 2000;76: 434-42.

\section{Comparing asthma prevalence estimates in Recife}

$\longrightarrow$

Dear Sir,

We would like to comment on a number of methodological issues related to an article by Britto et al. ${ }^{1}$ recently published in this journal.

One of the objectives of that study was to evaluate the diagnostic accuracy of the annual prevalence of wheezing as an indicator of asthma. To do this the authors compared answers to two different questions: question 2 (Q2) of the core asthma module of the ISAAC questionnaire - "Have you had wheezing in the past 12 months"? and question 6 (Q6) -"Have you ever had asthma?". It has been previously suggested that, in the absence of a gold standard, results obtained by administering a questionnaire of the signs and symptoms of asthma can be compared to documented diagnosis of asthma made by physicians in the same patients. ${ }^{2}$ In our opinion, information obtained through Q6 cannot be taken as equivalent to a history of physiciandiagnosed asthma (clinical examination and diagnosis made by a health professional), since participants' replies to this question will be determined by their own understanding of the term 'asthma' rather than by an objective measure of the presence of that disease. Therefore, the reported information seems to merely represent data on the agreement between answers to two separate questions rather than information on the validation of Q2. Validating this question would have required the comparison of replies to Q2 with results from either an objective test (e.g. lung function test), or a clinical examination by a physician, or documented information on a previous diagnosis of asthma from medical records. ${ }^{2}$
A second methodological issue is concerned with the use of the term cansaço (which in English means feeling breathless or short of breath) as part of the translation of the term "wheeze". Although the ISAAC study group had suggested that asthmatic children and their parents could be asked to describe breathing patterns during an asthma episode, ${ }^{3}$ we think that the translation of the term "wheeze" as "cansaço" used in the present study might not be appropriate. First, the term "wheeze" included in the core module of the ISAAC questionnaire corresponds to the terms "sibilos", "piado" or "chiado", in Brazilian Portuguese. In contrast, the term "cansaço" (shortness of breath) has a broader meaning and, in the Brazilian context, it is frequently associated with several clinical conditions other than asthma. Second, the English version of the questionnaire that was used in phase I of the ISAAC only included the terms "wheeze", "cough" and "asthma" (and not "breathless" or "short of breath"). 4 The term "breathless" or "short of breath" was only introduced later in the English version of the phase II ISAAC core questionnaire (module Wheeze and Breathlessness Supplementary Questionnaire). ${ }^{3}$ It is worth noting that the term "cansaço" did also not appear in the Brazilian version of the questionnaire designed to be used in Phase I of the ISAAC in Brazil. ${ }^{5}$ Finally, other three English versions of questionnaires designed to study respiratory diseases have used the terms "wheeze", "breathless" and "short of breath" in separate questions or as "shortness of breath with wheezing" (IUATLD, ATS and MRC). ${ }^{2}$ And it has been shown that questions that use the terms "breathless" and "short of breath" have lower specificity in correctly identifying asthma than those using the term "wheeze". 2

As a result, by accepting the term "cansaço" as a translation of "wheeze", Britto et al. may have obtained higher prevalence estimates than surveys based on questionnaires that did not include that term, making the results of the present study less comparable. Moreover, it is unclear whether the term "cansaço" was used in the survey conducted in $1994-1995^{6}$ or only in the 2000 survey and, if it was not used, interpretation of the findings from this comparative study will be difficult. In conclusion, we would like to suggest that future surveys of this type use standard questionnaires (e.g. ISAAC) without modification in order to preserve comparability of results across countries and over time. If modifications are judged necessary, they should be incorporated as additional questions, allowing separate analyses, as recommended in textbooks. ${ }^{7}$

\section{Sérgio Souza da Cunha}

MD, PhD. Projeto Fatores de risco para Asma e Alergia em Salvador, Instituto de Saúde Coletiva, Universidade Federal da Bahia (UFBA), Salvador, BA

E-mail: cunhass@ufba.br

\section{Mar Pujades Rodríguez}

MD, DTM, MSc. School of Community Health Sciences,

Nottingham University, United Kingdom

E-mail: mcxmdmp@nottingham.ac.uk

\section{References}

1. Britto MC, Bezerra PG, Brito RC, Rego JC, Burity EF, Alves JG. Asma em escolares do Recife - comparação de prevalências: 1994-95 e 2002 (Asthma in schoolchildren from Recife, Brazil, Prevalence comparison: 1994-95 and 2002). J Pediatr (Rio J). 2004;80:391-400. 